\title{
Filme ativo de acetato de celulose incorporado com nanosuspensão de curcumina
}

\section{Active film of cellulose acetate incorporated with nanosuspension of curcumin}

\author{
Deivis de Moraes Carvalho ${ }^{1 *}$, Katiuchia Pereira Takeuchi², Robson Maia Geraldine', \\ Celso José de Moura ${ }^{1}$ e Miriam Fontes Araujo Silveira ${ }^{1}$ \\ 'Departamento de Engenharia de Alimentos, Escola de Agronomia, \\ Universidade Federal de Goiás - UFG, Goiânia, GO, Brasil \\ ${ }^{2}$ Departamento de Alimentos e Nutrição, Faculdade de Nutrição, \\ Universidade Federal de Mato Grosso - UFMT, Cuiabá, MT, Brasil \\ *deivism.carvalho@gmail.com
}

\begin{abstract}
Resumo
As embalagens ativas podem liberar substâncias para inibir a microbiota e as reações na superfície dos alimentos. Dentre essas substâncias a curcumina pode ser usada pois apresenta atividade antimicrobiana e antioxidante. A difusão de compostos ativos em filmes de acetato de celulose pode ser estendida por longos períodos a taxa controlada. Sua adição pode afetar as propriedades mecânicas e de barreira dos filmes, tornando-se necessário mensurar estas alterações. Assim, objetivou-se preparar filmes ativos de acetato de celulose e tween 80 incorporados com mistura simples de curcumina e nanosuspensão de curcumina e avaliar cor, propriedades mecânicas, permeabilidade ao $\mathrm{CO}_{2}$ e migração do componente ativo. A curcumina influenciou as características mecânicas, ópticas e não afetou a permeabilidade dos filmes. O tween 80 favoreceu a migração da curcumina e a redução do tamanho das partículas não aumentou a migração de curcumina.
\end{abstract}

Palavras-chave: curcumina, embalagem ativa, migração, nanosuspensão.

\begin{abstract}
Active packaging can release substances to inhibit microbiota and reactions on the surface of foods; among these substances, curcumin can be applied for its antimicrobial and antioxidant activity. The diffusion of active compounds in cellulose acetate films can be extended for long periods at controlled rate. The addition may affect the mechanical and barrier properties of films, requiring the measurement of the alterations. The purpose of this study was to prepare active films of cellulose acetate and tween 80 incorporated with a simple mixture of curcumin and curcumin nanosuspension as well as assess color, mechanical properties, permeability to $\mathrm{CO}_{2}$ and migration of the active component. Curcumin influenced the mechanical and optical characteristics of the films but had no effect on the permeability to $\mathrm{CO}_{2}$. Tween 80 favored the migration, and the reduction in particles size did not increase the migration of curcumin.
\end{abstract}

Keywords: curcumin, active packaging, migration, nanosuspension.

\section{Introdução}

Embalagens ativas podem inibir ou retardar a microbiota e as reações na superfície dos alimentos, onde normalmente se inicia a deterioração ${ }^{[1]}$. As embalagens ativas têm um grande potencial na indústria alimentícia para aumentar a vida de prateleira e a segurança dos alimentos, entre essas, encontram-se os filmes de acetato de celulose (AC) incorporados com agentes antideteriorantes, incluindo nanopartículas ${ }^{[2]}$.

Os derivados acetilados da celulose podem ser produzidos por reações de acetilação. Essas reações ocorrem geralmente com o uso de ácido acético como solvente, anidrido acético como agente acetilante e ácidos sulfúrico e perclórico como catalisadores. O AC é produzido pela substituição dos grupos hidroxila das unidades de glicose por grupos acetila $^{[3]}$. Os filmes são preparados por meio da solubilização de pellets de AC em acetona seguido de espalhamento sobre uma superfície lisa, formando uma fina camada de solução, que favorece a volatilização da acetona e a formação do filme (método casting) ${ }^{[4]}$. A etapa de solubilização dos pellets em acetona é o momento ideal para a incorporação de componentes ativos.

A adição de substâncias nanoparticuladas ou não afetam as propriedades mecânicas e de barreira dos filmes, quer essas alterações sejam desejáveis ou não. A presença de agentes tensoativos ou outros aditivos, utilizados para incorporar de forma eficiente o material no interior da 
matriz polimérica, pode também afetar a difusividade ou a solubilidade de permeantes ${ }^{[5]}$. A adição de carvacrol e timol ao polipropileno (PP), por exemplo, modificaram nos filmes a barreira ao oxigênio e as propriedades mecânicas ${ }^{[6]}$ e a adição de glicerol melhorou a dispersão de amido de arroz em polietileno de baixa densidade (PEBD) e, consequentemente, as suas propriedades de tração ${ }^{[7]}$. Diante disso, é necessário mensurar as possíveis alterações geradas no que tange às propriedades físicas, mecânicas e de barreira ${ }^{[8]}$.

A difusão de substâncias através de filmes de AC pode ser estendida por um período mais longo de tempo a uma taxa controlada e, assim, serem utilizados como membranas de controle de taxa para o desenvolvimento de sistemas de liberação de substâncias ${ }^{[9]}$. A curcumina é um composto amarelo-laranja produzido a partir dos rizomas de cúrcuma, que é amplamente usada como corante ${ }^{[10]}$. A curcumina e óleos essenciais de cúrcuma apresentam atividade antimicrobiana ${ }^{[11-13]}$ e antioxidante, podendo ser incorporados em filmes de AC. A curcumina foi um antioxidante eficaz em diferentes ensaios in vitro quando em comparação com compostos antioxidantes convencionais $^{[14]}$. Pode ser usada para minimizar ou prevenir oxidação lipídica de produtos farmacêuticos e alimentícios, retardando a formação de produtos de oxidação tóxicos, mantendo a qualidade nutricional e prolongando a vida de prateleira dos produtos $^{[14]}$.

Os objetivos desse trabalho foram preparar filmes ativos de AC incorporados com mistura simples de curcumina em tween 80 (MS) e com nanosuspensão de curcumina em tween 80 (NS) e avaliar cor, propriedades mecânicas, permeabilidade ao $\mathrm{CO}_{2}$ e migração do componente ativo.

\section{Material e Métodos}

\subsection{Preparação dos filmes}

Para produção dos filmes (Tabela 1) foram usados: Curcumina marca Sigma-Aldrich ref. C1386 lote 081M1611V; tween 80 P.A. marca Vetec Cód. 749 Lote 1103476; mistura simples preparada com $22,2 \%$ de curcumina em tween 80 ; nanosuspensão de curcumina preparada com $22,2 \%$ de curcumina em tween 80 produzida conforme Carvalho et al. ${ }^{[15]}$; acetona P.A. 99,6\% marca Neon ref. 02595 lote 10794; acetato de celulose cedido pela empresa Rhodia ${ }^{\circledR}$

Os filmes foram preparados pelo método "casting"[4]. Os materiais foram colocados em seus respectivos frascos de preparação, em quantidade suficiente para se obter filmes (MS, NS e CP) de AC com 1\% (p/p) de curcumina, ou filmes isentos de curcumina (T80). Foram adicionados em cada frasco $36 \mathrm{~mL}$ de acetona e $4 \mathrm{~g}$ de AC. Os frascos âmbar foram colocados em homogeneizador rotativo (Phoenix, modelo AP32, Araraquara) por $16 \mathrm{~h} \mathrm{e}$, em seguida, deixados em repouso por $2 \mathrm{~h}$, para evitar a formação de bolhas.

Tabela 1. Filmes produzidos.

\begin{tabular}{cc}
\hline Tratamentos & Siglas \\
\hline Filme com mistura simples & $\mathrm{MS}$ \\
Filme com nanosuspensão & $\mathrm{NS}$ \\
Filme apenas com curcumina & $\mathrm{CP}$ (controle para os testes de migração) \\
Filme apenas com tween 80 & $\mathrm{~T} 80$ (controle para as análises de cor, \\
& permeabilidade e propriedades mecânicas) \\
\hline
\end{tabular}

As soluções filmogênicas foram, então, vertidas em placas de vidro com superfície lisa e espalhadas manualmente com o auxílio de um bastão de vidro com regulagem de espessura nas extremidades. Após a evaporação da acetona, obtiveram-se filmes com espessura e gramatura definidos.

\subsection{Espessura e gramatura}

A espessura foi analisada através da medida dos valores de 5 pontos aleatórios em diferentes segmentos dos filmes, utilizando-se micrômetro com resolução de $0,0001 \mathrm{~mm}$ (Qualitylabor, modelo MEP/Q, Perus). As amostras que apresentaram espessura inferir a $38 \mu \mathrm{m}$ ou superior a $42 \mu \mathrm{m}$ ao longo da sua área foram descartadas. A gramatura foi feita de acordo com a norma ASTM-D646-96 $6^{[16]}$.

\subsection{Cor e opacidade}

Os filmes MS e NS foram analisados quanto à cor e opacidade, tendo como controle filme T80. A cor e a opacidade dos filmes foram determinadas utilizando-se um colorímetro de bancada (Hunter Associates Laboratory Inc, modelo Colorquest II, Reston). A avaliação foi feita através dos parâmetros $L^{*}, a^{*}$ e b*. Onde $\mathrm{L}^{*}$ representa a luminosidade (sendo $\mathrm{L}^{*}=0$ preto e $\mathrm{L}^{*}=100$ branco) e a* e b* a cromaticidade (sendo $+a^{*}$ vermelho, $-a^{*}$ verde, $+\mathrm{b}^{*}$ amarelo e $-\mathrm{b}^{*}$ azul $)^{[17]}$. Segundo Schanda ${ }^{[18]}$, um dos métodos mais utilizados para o cálculo de diferenças de cor é o $\Delta \mathrm{E}$, que usa os valores numéricos do modelo CIELAB, conforme a Equação 1, onde $\Delta \mathrm{L}^{*}, \Delta \mathrm{a}^{*} \mathrm{e} \Delta \mathrm{b}^{*}$ são os valores numéricos do modelo CIELAB entre duas cores medidas. Nesse trabalho o $\Delta \mathrm{E}^{*}$ foi calculado usando-se como parâmetro para $\mathrm{L}_{0}{ }^{*}, \mathrm{a}_{0}{ }^{*}$ e $\mathrm{b}_{0}{ }^{*}$ uma placa padrão de cor branca e $\mathrm{L}_{1}{ }^{*}$, $\mathrm{a}_{1}^{*} \mathrm{e} \mathrm{b}_{1}^{*}$ as amostras dos filmes.

$$
\begin{aligned}
& \Delta L^{*}=L_{1}{ }^{*}-L_{0}{ }^{*}, \Delta a^{*}=a_{1}{ }^{*}-a_{0}{ }^{*}, \Delta b^{*}= \\
& b_{1}{ }^{*}-b_{0}{ }^{*} \cdot \Delta E^{*}=\sqrt{\Delta L^{* 2}+\Delta a^{* 2}+\Delta b^{* 2}}
\end{aligned}
$$

As determinações de opacidade foram feitas, após a calibração do colorímetro com um fundo padrão branco, cinza e preto. A opacidade foi determinada através da Equação 2.

$$
O p=(100 * O p n) / O p b
$$

Onde:

Op $=$ opacidade do filme $(\%)$;

Opn $=$ opacidade do filme sobreposto a um fundo negro;

$\mathrm{Opb}=$ opacidade do filme sobreposto a um fundo branco.

\subsection{Propriedades mecânicas}

Os filmes MS e NS foram analisados quanto às propriedades mecânicas, tendo como controle filmes T80. Os testes de tração foram realizados em uma máquina universal de ensaios (Instron, modelo 3367, Grove City) com controle pneumático de tração e com capacidade para células de carga de até $30 \mathrm{kN}$ ou $6750 \mathrm{Lbs}$, de acordo com a norma ASTM-D882-10 ${ }^{[19]}$. Foram preparadas tiras de amostras com largura de $10 \mathrm{~mm}$, comprimento de $120 \mathrm{~mm}$ e espessura entre 38 e $42 \mu \mathrm{m}$. As amostras foram acondicionadas em envelopes de papel e climatizadas entre 45 e $55 \%$ de umidade relativa e temperatura de 21 a $25{ }^{\circ} \mathrm{C}$ por $48 \mathrm{~h}$. $\mathrm{O}$ equipamento foi ajustado com espaço entre ganchos de 
$100 \mathrm{~mm}$. As amostras foram tracionadas com velocidade de $12 \mathrm{~mm} . \mathrm{min}^{-1}{ }^{-1}$ utilizando-se no equipamento uma célula de carga com capacidade de 500 N. Analisaram-se a tensão máxima, tensão na ruptura, o alongamento ou extensão máxima e o módulo de elasticidade ou módulo de Young.

\subsection{Permeabilidade ao gás carbônico}

Os filmes MS e NS foram analisados quanto à permeabilidade ao $\mathrm{CO}_{2}$, tendo como controle filmes T80. A permeabilidade ao $\mathrm{CO}_{2}$ foi medida em um analisador PERME VAC - V1 (Labthink, China). A análise foi feita segundo a norma ASTM-D1434-82[20], com amostras de espessura entre 38 e $42 \mu \mathrm{m}$. Previamente, os filmes foram cortados em formato circular com $7 \mathrm{~cm}$ de diâmetro e acondicionados em dessecador com sílica por $48 \mathrm{~h}$ na temperatura de $25^{\circ} \mathrm{C}$.

\subsection{Migração da curcumina para óleo de soja}

Os filmes MS e NS foram analisados quanto à migração tendo os filmes CP como controle. As amostras dos filmes foram cortadas nas dimensões de 10 x $10 \mathrm{~cm}$, dobradas de maneira sanfonada (para expor toda a área de contato do filme) e colocadas em tubos de ensaio com capacidade de $50 \mathrm{~mL}$. Em seguida, foram adicionados aos tubos $25 \mathrm{~mL}$ de óleo de soja comercial refinado tipo 1, contendo antioxidante Terc-butil-hidroquinona e ácido cítrico. Os tubos foram tampados e cobertos com papel alumínio para evitar a exposição à luz. Posteriormente, foram colocados em homogeneizador rotativo (Phoenix, modelo AP32, Araraquara). Todo o procedimento foi feito em sala climatizada a $25^{\circ} \mathrm{C}$. A concentração de curcumina no óleo de soja foi determinada a cada 24 h, até a estabilização da migração. Foi utilizada metodologia baseada na NBR $13624^{[21]}$ com modificações na etapa de preparação das amostras e com a construção de uma curva analítica (absorbância/concentração). Durante as leituras em espectrofotômetro (Biospectro, modelo SP-220, Curitiba), uma amostra controle sem curcumina foi usada como "branco". As amostras foram analisadas em comprimento de onda de $425 \mathrm{~nm}$.

Conforme Takeuchi ${ }^{[22]}$, uma equação de cinética de primeira ordem (Equação 3) foi usada para ajustar os dados de migração de curcumina das diferentes amostras de filmes (CP, MS e NS) em função do tempo de contato com o óleo. Foram usados os resultados de concentração expressos em mg.L $\mathrm{L}^{-1}$.

$$
C(t)=C_{s s}+A \cdot \exp (-k . t)
$$

Onde: $\mathrm{C}(\mathrm{t})$ é a propriedade medida (concentração) como uma função do tempo, $\mathrm{C}_{\mathrm{ss}}$ é o valor da propriedade no estado estacionário (concentração no equilíbrio), t (minutos) é o tempo, k é a taxa de migração $\left(m g \cdot \mathrm{L}^{-1} \cdot \mathrm{min}^{-1}\right)$ e A é um parâmetro de ajuste.

\subsection{Análise estatística}

Os filmes foram feitos com 5 repetições e as análises realizadas em triplicata, com exceção dos testes de tração, que foram utilizadas 10 tiras de filme para cada repetição. A diferença entre os tratamentos foi avaliada pela análise de variância e o teste de Tukey $(\mathrm{P} \leq 0,05)$.

\section{Resultados e Discussão}

\subsection{Cor e opacidade}

A adição de curcumina produziu filmes amarelos translúcidos, porém mais opacos que o controle (Figura 1). Assim, houve diferença significativa na cor e na opacidade (Tabela 2) das amostras com curcumina (MS e NS) em relação às sem curcumina (T80). Pode-se observar que o parâmetro $\mathrm{L}^{*}$, referente a luminosidade, foi igual para as amostras com curcumina, diferenciando-se significativamente do controle. O parâmetro $\Delta \mathrm{E}^{*}$, que representa a diferença de cor entre as amostras, foi igual para MS e NS. A opacidade também foi influenciada pela pigmentação provocada pela curcumina, diferindo significativamente do fime controle. Porém, não houve diferença significativa entre os tratamentos com curcumina, mostrando que a redução do tamanho das partículas não influenciou os parâmetros analisados.

A alta solubilidade da curcumina em acetona fez com que a curcumina da nanosuspensão e da mistura simples se dispersasse da mesma maneira no polímero e como a concentração de curcumina foi a mesma nas duas preparações, os filmes não apresentaram diferença de cor.

\subsection{Propriedades mecânicas}

Os filmes analisados apresentaram espessura entre 38 e $42 \mu \mathrm{m}$ e gramatura média de 5,24 $\pm 0,04$ g.m $\mathrm{m}^{-2}$. Os resultados dos testes de tração encontram-se na Tabela 3.

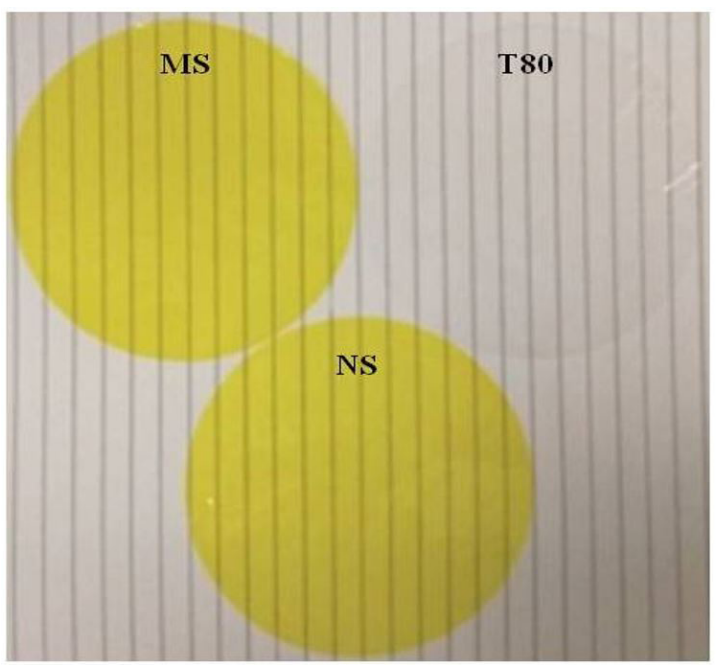

Figura 1. Filmes MS, NS e T80 (controle) de AC sobre fundo listrado.

Tabela 2. Valores das análises de cor e opacidade dos filmes.

\begin{tabular}{lccr}
\hline Tratamentos & $\mathbf{L}^{*}$ & $\Delta \mathbf{E}^{*}$ & Opacidade \\
\hline Filme T80 & $92,5 \pm 0,5^{\mathrm{a}}$ & $1,7 \pm 0,5^{\mathrm{a}}$ & $6,2 \pm 0,6^{\mathrm{a}}$ \\
Filme MS & $89,0 \pm 0,4^{\mathrm{b}}$ & $83,5 \pm 0,4^{\mathrm{b}}$ & $10,1 \pm 0,2^{\mathrm{b}}$ \\
Filme NS & $88,9 \pm 0,7^{\mathrm{b}}$ & $83,1 \pm 0,6^{\mathrm{b}}$ & $10,4 \pm 0,6^{\mathrm{b}}$ \\
\hline T80 - Tween & $80($ controle $), \mathrm{MS}-\mathrm{Mistura}^{\mathrm{S}}$ imples \\
NS - Nanosuspensão, L* - Luminosidade, $\Delta \mathrm{E}^{*}$ - Diferença de cor \\
no modelo CIELAB. Letras diferentes $(\mathrm{a}, \mathrm{b})$ nas colunas representam \\
diferença significativa ( $\leq 0,05)$.
\end{tabular}


Em todas as análises de tração, os filmes com curcumina (MS e NS) não apresentaram diferença significativa entre eles. Os valores do módulo de elasticidade não diferiram significativamente entre as amostras dos filmes MS, NS e T80 (controle). Na Figura 2 observa-se que as curvas apresentam praticamente a mesma inclinação até atingir a tensão máxima. $\mathrm{O}$ filme NS apresentou diferença significativa do filme T80 na avaliação de tensão máxima, tensão na ruptura e alongamento, enquanto o filme MS só apresentou diferença significativa do filme T80 quanto ao alongamento. Os resultados indicam que a adição de $1 \%$ de curcumina influenciou as características mecânicas dos filmes reduzindo significativamente o seu alongamento em ambos os tratamentos e a resistência à tração nos filmes NS.

A tração expressa a resistência do material à deformação por alongamento quando submetido a uma força longitudinal. Pode-se observar na Figura 2 que a deformação é o alongamento relativo da amostra em relação ao seu comprimento inicial. Primeiramente, o material oferece resistência crescente à tração, provocando alongamento. A partir de certo ponto, a resistência passa a ser menor para uma mesma taxa de deformação até o ponto de escoamento, onde é possível alongar o filme sem que haja aumento da resistência. Se continuar com o alongamento, o material suportará durante certo tempo, até que se rompa ${ }^{[23]}$.

Coelho $^{[24]}$ incorporou montmorilonita em PEBD com espessura média de $100 \mu \mathrm{m}$, e nos filmes controle (sem montmorilonita) obteve tensão máxima de 14,97 MPa e alongamento de aproximadamente $340 \%$. Observou também redução nos valores de tensão e alongamento com o aumento da adição de montmorilonita. Takeuchi ${ }^{[25]}$ trabalhou com filmes de AC incorporados com montmorilonita e com óleo resina de açafrão, com espessura de $40 \pm 5 \mu \mathrm{m}$ e corpos de prova com 17,5 $\mathrm{cm}$ de comprimento e 2,4 $\mathrm{cm}$ de largura. Para filmes sem aditivos obteve tensão na ruptura de $35,37 \mathrm{MPa}$, extensão máxima de $2,48 \%$ e módulo de elasticidade de $2328 \mathrm{MPa}$. Magalhães ${ }^{[26]}$ trabalhou com óleos essenciais incorporados em filmes de AC com espessura de $40 \pm 4 \mu \mathrm{m}$

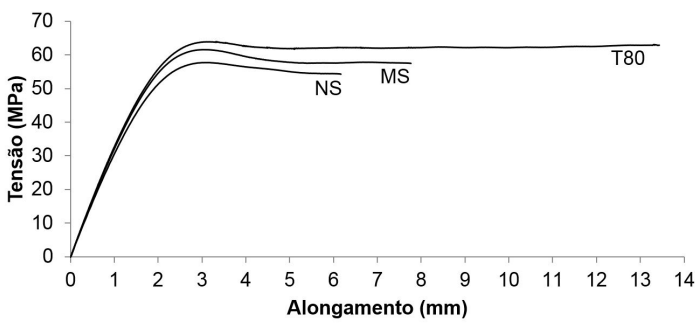

Figura 2. Representação gráfica da análise de tração dos filmes MS, NS e T80 (controle). e preparou os corpos de prova com $12 \mathrm{~cm}$ de comprimento e $1 \mathrm{~cm}$ de largura. A adição de óleos essenciais influenciou nas propriedades mecânicas. A tensão na ruptura variou de $60 \mathrm{MPa}$ (filmes controle) a $9 \mathrm{MPa}$ (fillmes com $50 \%$ de óleo). $\mathrm{O}$ alongamento nos filmes sem óleo foi de cerca de $10 \% \mathrm{e}$ com óleo variou entre 4 e $13 \%$. O módulo de elasticidade foi de aproximadamente $2800 \mathrm{MPa}$ para filmes controle e variou entre 500 e $2400 \mathrm{MPa}$ para os filmes com diferentes concentrações de óleo essencial. Comparando-se com os resultados do presente trabalho, verifica-se que o filme controle (T80) apresentou maior módulo de elasticidade, tensão na ruptura e alongamento que o filme de AC controle (sem tween 80 ) avaliado por Magalhães ${ }^{[26]}$. Pode-se então sugerir que o tween 80 na quantidade adicionada interagiu bem com a matriz polimérica sem prejudicar a estrutura dos filmes. A adição de curcumina reduziu significativamente o alongamento dos filmes MS e NS e a resistência dos filmes NS, assim como ocorreu com a montmorilonita e os óleos essenciais.

\subsection{Permeabilidade ao gás carbônico}

Observa-se que não houve diferença significativa na permeabilidade ao $\mathrm{CO}_{2}$ entre os filmes MS, NS e T80 (Tabela 4).

A permeabilidade dos filmes ao $\mathrm{CO}_{2}$ depende de muitos fatores, como o tipo de polímero utilizado, a substância e a quantidade adicionada ao polímero ${ }^{[27]}$. Ao estudarem filmes de polietileno e acetato de vinil etileno, Mousavi et al. ${ }^{[28]}$ verificaram que o aumento da espessura diminui a permeabilidade ao $\mathrm{CO}_{2}$ e $\mathrm{O}_{2}$. A espessura dos filmes no presente trabalho variou de 38 a 42 micrômetros, conforme a metodologia ${ }^{[20]}$, não oferecendo interferência significativa nos resultados. Gontard et al. ${ }^{[29]}$ descreveram que os plastificantes podem causar baixa coesão nos filmes, gerando uma estrutura menos eficiente como barreira. Entende-se que essa baixa coesão pode resultar de uma menor interação entre as moléculas do polímero e do plastificante proporcionando espaços por onde poderiam permear as moléculas dos gases. $\mathrm{O}$ filme controle de PEBD produzido por Coelho ${ }^{[24]}$, apresentou permeabilidade ao $\mathrm{CO}_{2}$ de aproximadamente $5800 \mathrm{~cm}^{3} \cdot \mathrm{m}^{-2} \cdot 24 \mathrm{~h} \cdot 0,1 \mathrm{MPa}$. O filme controle de AC obtido por Magalhães ${ }^{[26]}$, sem aditivos, apresentou permeabilidade de $6590 \mathrm{~cm}^{3} \cdot \mathrm{m}^{-2} \cdot 24 \mathrm{~h} .0,1 \mathrm{MPa}$ e os filmes incorporados com óleo apresentaram permeabilidade de 15588 e $23441 \mathrm{~cm}^{3} \cdot \mathrm{m}^{-2} \cdot 24 \mathrm{~h} \cdot 0,1 \mathrm{MPa}$. O autor afirmou que a adição de óleos essenciais nas concentrações de $15,25,35$ e $50 \%$ aumentaram a permeabilidade do filme de $\mathrm{AC}$ ao $\mathrm{CO}_{2}$. Nesse trabalho, a permeabilidade ao $\mathrm{CO}_{2}$ do filme T80 foi de $5598 \mathrm{~cm}^{3} \cdot \mathrm{m}^{-2} \cdot 24 \mathrm{~h} .0,1 \mathrm{MPa}$. Provavelmente, a adição de tween 80 na concentração utilizada $(3,5 \%)$ reduziu ou pelo menos não alterou a permeabilidade do filme. Pode-se sugerir que nessa concentração houve boa

Tabela 3. Valores das análises de tração dos filmes.

\begin{tabular}{lcccc}
\hline \multicolumn{1}{c}{ Tratamentos } & $\begin{array}{c}\text { Tensão Máxima } \\
(\mathbf{M P a})\end{array}$ & $\begin{array}{c}\text { Tensão na Ruptura } \\
(\mathbf{M P a})\end{array}$ & $\begin{array}{c}\text { Alongamento } \\
(\%)\end{array}$ & $\begin{array}{c}\text { Módulo de Elasticidade } \\
\text { (MPa) }\end{array}$ \\
\hline Filme T80 & $66,0 \pm 3,0^{\mathrm{a}}$ & $65,0 \pm 3,3^{\mathrm{a}}$ & $15,3 \pm 4,2^{\mathrm{a}}$ & $3399,4 \pm 25,7^{\mathrm{a}}$ \\
Filme MS & $63,1 \pm 3,4^{\mathrm{ab}}$ & $60,7 \pm 4,1^{\mathrm{ab}}$ & $7,2 \pm 1,3^{\mathrm{b}}$ & $3400,2 \pm 122,7^{\mathrm{a}}$ \\
Filme NS & $56,5 \pm 2,8^{\mathrm{b}}$ & $54,5 \pm 2,8^{\mathrm{b}}$ & $6,7 \pm 1,2^{\mathrm{b}}$ & $3245,7 \pm 102,3^{\mathrm{a}}$ \\
\hline
\end{tabular}

T80 - Tween 80 (controle), MS - Mistura Simples, NS - Nanosuspensão. Letras diferentes $(\mathrm{a}, \mathrm{b}$ ) nas colunas representam diferença significativa $(\mathrm{p} \leq 0,05)$. 
interação entre as moléculas do tween 80 e do polímero ou uma boa coesão na matriz polimérica, diferente do que ocorreu com os trabalhos comparados. Todos os filmes, incluindo o filme controle receberam a mesma quantidade de tween 80 .

\subsection{Migração da curcumina em óleo de soja}

A migração da curcumina dos filmes para o óleo de soja foi acompanhada durante 30 dias. A taxa de migração na primeira semana foi maior, e reduziu ao longo do tempo até cessar em torno de 22 dias de teste. Verificou-se que $0,06 \%$ da curcumina presente no filme $\mathrm{CP}$ (controle) migrou para o óleo, enquanto que $0,13 \%$ e $0,14 \%$ da curcumina dos filmes NS e MS, respectivamente, também migraram. Nos resultados apresentados na Tabela 5 verifica-se que a concentração (mg. $\left.\mathrm{L}^{-1}\right)$ de curcumina migrada para o óleo de soja, após alcançar o estado estacionário (Css), foi maior para os filmes MS e NS. Não houve diferença significativa na migração entre os filmes NS e MS. A taxa de migração (K) não apresentou diferença significativa para os filmes ativos incorporados com curcumina. A curcumina foi altamente solúvel na acetona usada na solução filmogênica, e independente de estar ou não nanoparticulada, ela foi totalmente solubilizada e dispersa na matriz polimérica. A curcumina aliada ao tween 80 (filmes MS e NS) agregouse da mesma maneira às cadeias poliméricas e difundiu-se na mesma concentração. A concentração no equilíbrio (Css) da curcumina migrada do filme CP foi menor, pois ela ficou mais presa ao polímero por não contar com a ajuda do dispersante para auxiliar na difusão.

Segundo Silveira ${ }^{[30]}$, que avaliou filmes de $\mathrm{AC}$ de 25 a $100 \mu \mathrm{m}$ de espessura incorporados com ácido sórbico, filmes de menor espessura apresentam índices de migração maiores devido a maior facilidade com que o solvente penetra na matriz dissolvendo parte do soluto e liberando-o para o solvente. Assim, foi importante o controle da espessura do material em análise.

Tabela 4. Permeabilidade dos filmes ao $\mathrm{CO}_{2}$.

\begin{tabular}{lc}
\hline Tratamentos & $\begin{array}{c}\text { Permeabilidade ao CO } \\
\left(\mathbf{c m}^{\mathbf{3}} \cdot \mathbf{m}^{-\mathbf{2}} \mathbf{2 4 h . 0 , 1 M P a}\right)\end{array}$ \\
\hline Filme T80 & $5598 \pm 498^{\mathrm{a}}$ \\
Filme MS & $5594 \pm 809^{\mathrm{a}}$ \\
Filme NS & $5011 \pm 614^{\mathrm{a}}$ \\
\hline
\end{tabular}

T80 - Tween 80 (controle), MS - Mistura Simples, NS - Nanosuspensão. Letras iguais (a) na coluna representam que não houve diferença significativa $(\mathrm{p}>0,05)$.

Tabela 5. Parâmetros da Equação 3 obtidos para os diferentes tratamentos dos filmes de AC incorporados com curcumina.

\begin{tabular}{lcccc}
\hline Tratamentos & $\begin{array}{c}\text { Css } \\
\left(\mathbf{m g} \cdot \mathbf{L}^{-1}\right)\end{array}$ & $\mathbf{A}$ & $\mathbf{K .}\left(\mathbf{1 0}^{\mathbf{- 4}} \mathbf{\text { mg.L }}\right.$ & \\
\hline Filme CP & $0,124932^{\mathrm{a}}$ & $-0,118160$ & $0,90^{\mathrm{a}}$ & $\mathbf{r}^{\mathbf{2}}$ \\
Filme MS & $0,284350^{\mathrm{b}}$ & $-0,251178$ & $0,82^{\mathrm{a}}$ & 0,98 \\
Filme NS & $0,249648^{\mathrm{b}}$ & $-0,210172$ & $1,04^{\mathrm{a}}$ & 0,98 \\
\hline
\end{tabular}

CP - Curcumina pura (controle), MS - Mistura simples, NS - Nanosuspensão, Css - Concentração no equilíbrio, A-Parâmetro matemático de ajuste, $\mathrm{K}$ - Velocidade de migração, $\mathrm{r}^{2}$ - Coeficiente de determinação. Letras diferentes $(a, b)$ nas colunas representam diferença significativa $(\mathrm{p} \leq 0,05)$.
Em uma embalagem ativa antioxidante, quando o antioxidante atinge o máximo da migração, ele começa a diminuir devido o seu consumo por aprisionamento de radicais livres a partir dos ácidos graxos submetidos ao processo de oxidação ${ }^{[31]}$. Para evitar que a curcumina fosse consumida durante o teste de migração, interferindo nos resultados, usou-se uma marca de óleo de soja refinado comercial já adicionado de antioxidante, ao abrigo da luz e do calor.

As Figuras 3, 4 e 5 representam as concentrações (mg.L-1 $\left.{ }^{-1}\right)$ experimentais e preditas de curcumina que migraram das amostras dos filmes CP, MS e NS para o óleo de soja, segundo a Equação 3.

Existem na literatura exemplos de embalagens ativas adicionadas de antioxidantes que obtiveram sucesso na prevenção da oxidação de alimentos. Polímeros incorporados com $\alpha$-tocoferol aumentaram a estabilidade oxidativa do óleo de milho de 12 para 16 semanas $^{[32]}$. Espera-se que filmes de AC incorporados com curcumina possam ser usados em embalagens ativas para prevenir a contaminação microbiana e a oxidação em alimentos sem oferecer risco para o consumidor. Recomendam-se mais estudos com outras concentrações de curcumina e tween 80 e com a aplicação do filme como embalagem em alimentos.

Do ponto de vista de risco para a saúde, a embalagem ativa pode ser considerada como segura se a migração do componente ativo for menor que os limites estabelecidos

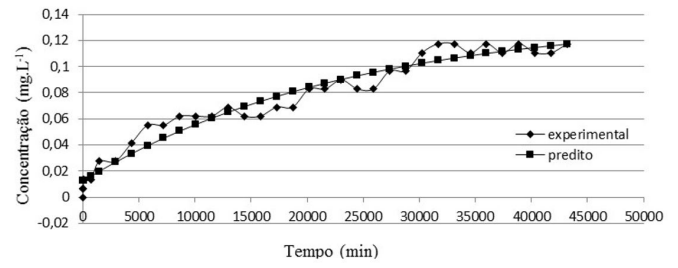

Figura 3. Migração da curcumina dos filmes CP $\left(\mathrm{mg} \cdot \mathrm{L}^{-1} \cdot \mathrm{min}^{-1}\right)$. Os valores preditos foram ajustados pela Equação 3.

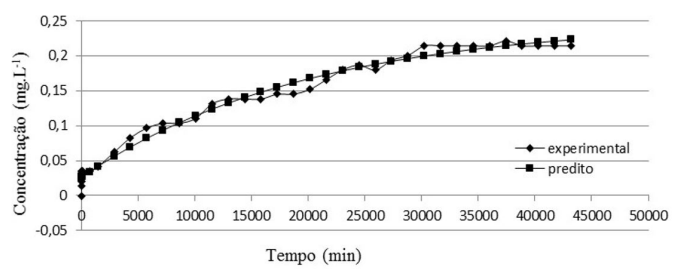

Figura 4. Migração da curcumina dos filmes MS $\left(\mathrm{mg} \cdot \mathrm{L}^{-1} \cdot \mathrm{min}^{-1}\right)$. Os valores preditos foram ajustados pela Equação 3 .

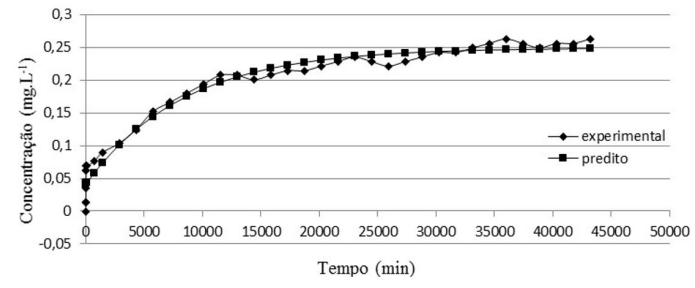

Figura 5. Migração da curcumina dos filmes NS $\left(m g \cdot \mathrm{L}^{-1} \cdot \mathrm{min}^{-1}\right)$. Os valores preditos foram ajustados pela Equação 3. 
pela legislação ${ }^{[33]}$. A curcumina é permitida para uso como aditivo em alimentos e está inscrita no Sistema Internacional de Numeração de Aditivos Alimentares do Codex Alimentarius, sob o número INS $100 \mathrm{i}^{[34-36]}$. A massa de curcumina migrada nesse estudo está abaixo dos limites máximos para ser usada como aditivo em alimentos, portanto não oferece risco. Exemplos: embutidos $\left(0,002\right.$ g. $\left.100 \mathrm{~g}^{-1}\right)$, bolos $\left(0,02 \mathrm{~g} .100 \mathrm{~g}^{-1}\right)$, balas $\left(0,015\right.$ g. $\left.100 \mathrm{~g}^{-1}\right)$, sopas $\left(0,005\right.$ g. $\left.100 \mathrm{~g}^{-1}\right)$, entre outros.

\section{Conclusões}

A adição de 1\% de curcumina influenciou as características mecânicas dos filmes, reduzindo a sua extensão máxima e a resistência à tração, mas não afetou a permeabilidade ao gás carbônico. A curcumina coloriu os filmes de amarelo, reduzindo o valor de $\mathrm{L}^{*}$ e aumentando $\Delta \mathrm{E}^{*}$ e a opacidade. Não houve diferença de coloração e de opacidade entre os filmes MS e NS. Os filmes MS e NS tiveram migração duas vezes maior que os filmes $\mathrm{CP}$ (controle) e não houve diferença na taxa de migração entre MS e NS. O tween 80 favoreceu a migração da curcumina. A alta solubilidade da curcumina em acetona não permitiu que a redução do tamanho das partículas contribuísse para uma diferente taxa de migração da curcumina.

\section{Agradecimentos}

Ao apoio financeiro através dos Editais: 04/CII-2008 - Rede Nanobiotec-Brasil Desenvolvimento e Avaliação de Embalagens Ativas Incorporadas com Nanobiocompostos para o Acondicionamento de Alimentos e Encomenda MCT/SEBRAE/FINEP, Plano de Trabalho No 04/2007 - Cooperação ICT's/MPE's, inserida em APL's - No 01/2007: Potencializando o açafrão de Mara Rosa-GO.

\section{Referências}

1. Devlieghere, F., Vermeiren, L., \& Debevere, J. (2004). New preservation technologies: possibilities and limitations. International Dairy Journal, 14(4), 273-285. http://dx.doi. org/10.1016/j.idairyj.2003.07.002.

2. Soares, N. F. F., Silva, W. A., Medeiros, E. A. A., Carelli, R. T., \& Espitia, P. J. P. (2011). Active packaging: new technologies for shelf-life extension. Italian Journal of Food Science, (supl.), 88. Recuperado em 21 de janeiro de 2014, de http://connection. ebscohost.com/c/articles/87765911/active-packaging-newtechnologies-shelf-life-extension.

3. Cerqueira, D. A., Rodrigues, G. R., Fo., Carvalho, R. A., \& Valente, A. J. M. (2010). Caracterização de acetato de celulose obtido a partir do bagaço de cana-de-açúcar por ${ }^{1} \mathrm{H}-\mathrm{RMN}$. Polimeros: Ciência e Tecnologia, 20(2), 85-91. http://dx.doi. org/10.1590/S0104-14282010005000017.

4. Soares, N. F. F. (1998). Bitterness reduction in citrus juice through naringinase immobilized into polymer film (Doctoral thesis). Cornell University, New York.

5. Duncan, T. V. (2011). Applications of nanotechnology in food packaging and food safety: Barrier materials, antimicrobials and sensors. Journal of Colloid and Interface Science, 363(1), 1-24. PMid:21824625. http://dx.doi.org/10.1016/j.jcis.2011.07.017.

6. Ramos, M., Jiménez, A., Peltzer, M., \& Garrigós, M. C. (2012). Characterization and antimicrobial activity studies of polypropylene films with carvacrol and thymol for active packaging. Journal of Food Engineering, 109(3), 513-519. http://dx.doi.org/10.1016/j.jfoodeng.2011.10.031.

7. Wang, Y. J., Liu, W., \& Sun, Z. (2004). Effects of glycerol and PE-g-MA on morphology, thermal and tensile properties of LDPE and rice starch blends. Journal of Applied Polymer Science, 92(1), 344-350. http://dx.doi.org/10.1002/app.20015.

8. Batista, J. A., Tanada-palmu, P. S., \& Grosso, C. R. F. (2005) Efeito da adição de ácidos graxos em filmes à base de pectina. Ciência e Tecnologia de Alimentos, 25(4), 781-788. http:// dx.doi.org/10.1590/S0101-20612005000400025.

9. Rao, P. R., \& Diwan, P. V. (1997). Permeability studies of cellulose acetate free films for transdermal use: Influence of plasticizers. Pharmaceutica Acta Helvetiae, 72(1), 47-51. PMid:9063088. http://dx.doi.org/10.1016/S0031-6865(96)00060-X.

10. Chattopadhyay, I., Biswas, K., Bandyopadhyay, U., \& Banerjee, R. K. (2004). Turmeric and curcumin: biological actions and medicinal applications. Current Science, 87(1), 44-53. Recuperado em 21 de janeiro de 2014, de http://repository. ias.ac.in/5196/.

11. Kamazeri, T. S., Samah, O. A., Taher, M., Susanti, D., \& Qaralleh, H. (2012). Antimicrobial activity and essential oils of Curcuma aeruginosa, Curcuma mangga, and Zingiber cassumunar from Malaysia. Asian Pacific Journal of Tropical Medicine, 5(3), 202-209. PMid:22305785. http://dx.doi. org/10.1016/S1995-7645(12)60025-X.

12. Al-Reza, S. M., Rahman, A., Parvin, T., Rahman, M. M., \& Rahman, M. S. (2011). Chemical composition and antibacterial activities of essential oil and organic extracts of curcuma aromatica salisb. Journal of Food Safety, 31(4), 433-438. http://dx.doi.org/10.1111/j.1745-4565.2011.00318.x.

13. Bhawana, B. L., Basniwal, R. K., Buttar, H. S., Jain, V. K., \& Jain, N. (2011). Curcumin nanoparticles: preparation, characterization, and antimicrobial study. Journal of Agricultural and Food Chemistry, 59(5), 2056-2061. PMid:21322563. http:// dx.doi.org/10.1021/jf104402t.

14. Ak, T., \& Gülçin, İ. (2008). Antioxidant and radical scavenging properties of curcumin. Chemico-Biological Interactions, 174(1), 27-37. PMid:18547552. http://dx.doi.org/10.1016/j. cbi.2008.05.003.

15. Carvalho, D. M., Takeuchi, K. P., Geraldine, R. M., Moura, C. J., \& Torres, M. C. L. (2015). Production, solubility and antioxidant activity of curcumin nanosuspension. Food Science and Technology, 35(1), 115-119. http://dx.doi.org/10.1590/1678457X.6515.

16. American Society for Testing and Material-ASTM. (2007). ASTM D 646-96. Standard test method for grammage of paper and paperboard (mass per unit area). West Conshohocken: ASTM.

17. Pehlivan, H., Balköse, D., Ülkü, S., \& Tihminliog `lu, F. (2005). Characterization of pure and silver exchanged natural zeolite filled poplypropylene composite films. Composites Science and Technology, 65(13), 2049-2058. http://dx.doi.org/10.1016/j. compscitech.2005.04.011.

18. Schanda, J. (2007). Colorimetry: understanding the CIE system. New Jersey: John Wiley \& Sons.

19. American Society for Testing and Material-ASTM. (2010). ASTM D 882-10: standard test method for tensile properties of thin plastic sheeting. West Conshohocken: ASTM.

20. American Society for Testing and Material-ASTM. (2009). ASTM D 1434-82: standard test method for determining gas permeability characteristics of plastic film and sheeting. West Conshohocken: ASTM.

21. Associação Brasileira de Normas Técnicas - ABNT. (1996). NBR 13624. Cúrcuma - determinação do teor de curcumina. Rio de Janeiro: ABNT. 
22. Takeuchi, K. P. (2008). Propriedades macro- e microscópicas de géis de proteinas do leite e $\kappa$-carragena (Tese de doutorado). Universidade Estadual de Campinas, Campinas.

23. Oliveira, L. M. D., Alves, R. M. V., Sarantópoulos, C. I. G. L., Padula, M., Garcia, E. E. C., \& Coltro, L. (1996). Ensaios para avaliação de embalagens plásticas flexiveis. Campinas: ITAL, CETEA.

24. Coelho, L. B. (2013). Efeito da incorporação do nanocomposto montmorilonita e do óleo essencial de orégano em filmes de polietileno de baixa densidade (Dissertação de mestrado). Universidade Federal de Goiás, Goiânia.

25. Takeuchi, A. P. (2012). Caracterização antimicrobiana de componentes do açafrão (curcuma longa l.) e elaboração de filmes ativos com montimorilonita e óleo resina de açafrão (Dissertação de mestrado). Universidade Federal de Goiás, Goiânia.

26. Magalhães, R. M. F. (2012). Atividade antimicrobiana de óleos essenciais e incorporação em filme de acetato de celulose na conservação de carne resfriada de frango (Dissertação de mestrado). Universidade Federal de Goiás, Goiânia.

27. Pinheiro, A. C. A., Cerqueira, M. A., Souza, B. W. S., Martins, J. T., Teixeira, J. A., \& Vicente, A. A. (2010). Utilização de revestimentos/filmes edíveis para aplicações alimentares. Boletim de Biotecnologia, 18-28. Recuperado em 21 de janeiro de 2014 de http://hdl.handle.net/1822/16725.

28. Mousavi, S. A., Gholizadeh, M., Sedghi, S., Pourafshari-Chenar, M., Barmala, M., \& Soltani, A. (2010). Effects of preparation conditions on the morphology and gas permeation properties of polyethylene (PE) and ethylene vinyl acetate (EVA) films. Chemical Engineering Research \& Design, 88(12), 1593-1598. http://dx.doi.org/10.1016/j.cherd.2010.03.013.

29. Gontard, N., Guilbert, S., \& Cuq, J. L. (1993). Water and glycerol as plasticizers affect mechanical and water vapor barrier properties of and edible wheat gluten film. Journal of Food Science, 58(1), 206-211. http://dx.doi.org/10.1111/j.1365-2621.1993. tb03246.x.
30. Silveira, M. F. A. (2005). Filme antimicrobiano de acetato de celulose incorporado com ácido sórbico na conservação de massa de pastel (Tese de doutorado). Universidade Federal de Viçosa, Viçosa.

31. Granda-Restrepo, D. M., Soto-Valdez, H., Peralta, E., Troncoso-Rojas, R., Vallejo-Córdoba, B., Gámez-Meza, N., \& Graciano-Verdugo, A. Z. (2009). Migration of $\alpha$-tocopherol from an active multilayer film into whole milk powder. Food Research International, 42(10), 1396-1402. http://dx.doi. org/10.1016/j.foodres.2009.07.007.

32. Graciano-Verdugo, A. Z., Soto-Valdez, H., Peralta, E., CruzZárate, P., Islas-Rubio, A. R., Sánchez-Valdes, S., SánchezEscalante, A., González-Méndez, N., \& González-Ríos, H. (2010). Migration of a-tocopherol from LDPE films to corn oil and its effect on the oxidative stability. Food Research International, 43(4), 1073-1078. http://dx.doi.org/10.1016/j. foodres.2010.01.019.

33. Tovar, L., Salafranca, J. S., Sãnchez, C., \& Nerín, C. (2005). Migration studies to assess the safety in use of a new antioxidant active packaging. Journal of Agricultural and Food Chemistry, 53(13), 5270-5275. PMid:15969507. http://dx.doi.org/10.1021/ jf050076k.

34. Codex Alimentarius Commission - CAC. (2015). CAC/GL 36-1989. Class names and the international numbering system for food additives. Roma: CAC.

35. Codex Alimentarius Commission-CAC. (2015). CAC/MISC 6-2015. List of codex specifications for food additives. Roma: CAC.

36. Codex Alimentarius Commission - CAC. (2009). CODEX STAN 256-2007. Standard for fat spreads and blended spreads. Roma: CAC.

Enviado: Jun. 19, 2015

Revisado: Nov. 09, 2015

Aceito: Mar: 11, 2016 